PROCEEDINGS OF THE

AMERICAN MATHEMATICAL SOCIETY

Volume 132, Number 10, Pages 2945-2950

S 0002-9939(04)07148-5

Article electronically published on June 2, 2004

\title{
THE STABLE RANK OF FULL CORNERS IN C*-ALGEBRAS
}

\author{
BRUCE BLACKADAR
}

(Communicated by David R. Larson)

\begin{abstract}
We give a treatment of Rieffel's theory of stable rank for C*algebras in terms of left invertibility of generalized nonsquare matrices, and prove that if $p$ is a full projection in a unital $\mathrm{C}^{*}$-algebra $A$, then the stable rank of the corner $p A p$ is at least as large as the stable rank of $A$.
\end{abstract}

\section{INTRODUCTION}

An algebraic theory of stable rank in rings was developed by H. Bass Bas68, primarily to handle cancellation problems in algebraic $K$-theory. M. Rieffel adapted the theory to $\mathrm{C}^{*}$-algebras (and more general topological algebras) Rie83. This theory was formally modeled on dimension theory for compact Hausdorff spaces, but it was quickly realized that stable rank does not resemble a dimension theory very closely in the noncommutative case. The theory has nonetheless proved interesting and useful, particularly with regard to nonstable $K$-theory questions (cf. [War80.)

Several variations of the theory of stable rank have been developed, such as the theory of real rank of [BP91]. Another variation, which behaves more like a true dimension theory for noncommutative $\mathrm{C}^{*}$-algebras, is the completely positive rank of [Win01].

The principal reason that stable rank does not behave like a dimension theory in the noncommutative case, and also the reason it gives nonstable $K$-theory information, is its behavior under forming matrix algebras ([Vn71], [Rie83, 6.1]): the stable rank $\operatorname{sr}\left(M_{n}(A)\right)$ is roughly $\operatorname{sr}(A)$ divided by $n$ (see 4.4 for the precise formula). In particular, $\operatorname{sr}\left(M_{n}(A)\right) \leq \operatorname{sr}(A)$. Since $A \cong p M_{n}(A) p$ for a "rank-one" projection $p$ (such a subalgebra is called a corner), this result suggests that the stable rank of any full corner (not contained in a proper two-sided ideal) $p A p$ in a unital $\mathrm{C}^{*}$-algebra $A$ satisfies $\operatorname{sr}(p A p) \geq \operatorname{sr}(A)$. This relationship was conjectured at the time of Rieffel's paper nearly twenty years ago, but only partial results have been known (see e.g. [Bla83 A6]).

In this paper, we give an exposition of the basics of the theory of stable rank which differs somewhat from that of Rie83, and which leads to a positive resolution (4.5) of this "loose end" from the theory, the main new result of the paper. The proofs are for the most part straightforward adaptations of arguments from Rie83.

Received by the editors November 20, 2002.

2000 Mathematics Subject Classification. Primary 46L05; Secondary $19 B 10$.

Key words and phrases. C*-algebra, stable rank.

This work was supported by NSF grant DMS-0070763. 


\section{BASIC DEFINITIONS}

For the convenience of the reader, we recall some standard definitions and facts about stable rank from Rie83.

Both stable rank and real rank are motivated by the following fact from topology ([HW41, VI.1], [Pea75, 3.3.2]; cf. Kat50 and [GJ60] for related results).

Theorem 2.1. Let $X$ be a compact metrizable space. Then all dimension theories coincide on $X$, and $\operatorname{dim}(X)$ can be characterized as the smallest $n$ with the following property: any continuous function $f: X \rightarrow \mathbb{R}^{n+1}$ can be uniformly approximated arbitrarily closely by $g: X \rightarrow \mathbb{R}^{n+1}$ such that $g(X)$ does not contain the origin in $\mathbb{R}^{n+1}$

Definition 2.2. Let $A$ be a unital $\mathrm{C}^{*}$-algebra. Let $\operatorname{Lg}_{n}(A)$ be the set of $\left(x_{1}, \ldots, x_{n}\right)$ in $A^{n}$ such that there exists $\left(y_{1}, \ldots, y_{n}\right) \in A^{n}$ with $\sum_{i=1}^{n} y_{i} x_{i}=1$.

The stable rank of $A$, denoted $\operatorname{sr}(A)$, is the smallest $n$ such that $L g_{n}(A)$ is dense in $A^{n}$. If there is no such $n$, set $\operatorname{sr}(A)=\infty$.

If $A$ is nonunital, then $\operatorname{sr}(A)$ is defined to be $\operatorname{sr}(\tilde{A})$.

The elements of $\operatorname{Lg}_{n}(A)$ are the $n$-tuples which generate $A$ as a left $A$-module. It is easily seen that $\left(x_{1}, \ldots, x_{n}\right) \in L g_{n}(A)$ if and only if $\sum_{i=1}^{n} x_{i}^{*} x_{i}$ is invertible. In particular, if $A=C(X)$, then $\left(f_{1}, \ldots, f_{n}\right) \in L g_{n}(A)$ if and only if for every $t \in X$ there is an $i$ such that $f_{i}(t) \neq 0$. Thus, since $\left(f_{1}, \ldots, f_{n}\right) \in A^{n}$ can be regarded as a continuous function from $X$ to $\mathbb{C}^{n} \cong \mathbb{R}^{2 n}$, we obtain from 2.1 that $s r(C(X))=\left\lfloor\frac{\operatorname{dim}(X)}{2}\right\rfloor+1$, where $\lfloor\cdot\rfloor$ denotes "integer part of."

The number $\operatorname{sr}(A)$ defined in Definition 2.2 is properly called the left topological stable rank of $A$, denoted $\operatorname{ltsr}(A)$ in Rie83; $R g_{n}(A)$ and $r t s r(A)$ can be defined analogously. Because of the involution, there is an obvious correspondence between $L g_{n}(A)$ and $R g_{n}(A)$, and $\operatorname{ltsr}(A)=\operatorname{rtsr}(A)$; this number is called $\operatorname{tsr}(A)$ in [Rie83] to distinguish it from the Bass stable rank $\operatorname{Bsr}(A)$. The topological stable rank of a $\mathrm{C}^{*}$-algebra was shown to coincide with the Bass stable rank in [HV84]; thus we may use the term "stable rank" and the notation $\operatorname{sr}(A)$ unambiguously.

\section{THE GENERALIZED MATRIX PICTURE}

Elements of $A^{n}$ may be regarded as $n \times 1$ matrices over $A$, and $L g_{n}(A)$ becomes the set of left invertible $n \times 1$ matrices, so stable rank can be defined in terms of density of left invertible matrices.

If $p$ and $q$ are projections in a $\mathrm{C}^{*}$-algebra $A$, it is useful to think of the subspace $p A q$ of $A$ as a space of "nonsquare matrices" with " $p$ rows" and " $q$ columns". If $r$ and $s$ are other projections orthogonal to $p$ and $q$ respectively, then a " $(p+r) \times(q+s)$ matrix" (an element of $(p+r) A(q+s)$ ) may be symbolically written as a $2 \times 2$ "block matrix": write $x \in(p+r) A(q+s)$ as

$$
\left[\begin{array}{ll}
p x q & p x s \\
r x q & r x s
\end{array}\right] .
$$

The algebraic operations in these sets (as subsets of $A$ ) can be calculated by formal matrix algebra.

It is convenient to generalize the notion of left invertibility:

Definition 3.1. Let $A$ be a $\mathrm{C}^{*}$-algebra, and let $p$ and $q$ be projections in $A$. An element $x \in p A q$ is left invertible (in $p A q$ or with respect to $(p, q)$ ) if there is $y \in q A p$ 
with $y x=q$. We write $L g_{(p, q)}(A)$ for the set of left invertible elements of $p A q$. If $p$ and $q$ are projections in $M_{n}(A)$, define $L g_{(p, q)}(A)$ to be $L g_{(p, q)}\left(M_{n}(A)\right)$.

It is easily seen that $x \in p A q$ is left invertible in $p A q$ if and only if $x^{*} x$ is invertible in $q A q$. Thus left invertibility with respect to $(p, q)$ really depends only on the $q$, and if $r \perp p$ and $L g_{(p, q)}(A)$ is dense in $p A q$, then $L g_{(p+r, q)}(A)$ is dense in $(p+r) A q$.

Recall that if $p$ and $q$ are projections in $A$, then $p$ and $q$ are (Murray-von Neumann) equivalent (in $A$ ), written $p \sim q$, if there is a $u \in A$ with $u^{*} u=p, u u^{*}=q$; $q$ is subordinate to $p$ (in $A$ ), written $q \precsim p$, if $q \sim q^{\prime} \leq p$, i.e. if there is a $u \in A$ with $u^{*} u=q$ and $u u^{*} \leq p$. We will write $n \cdot q \precsim p$ if there are mutually orthogonal subprojections $p_{1}, \ldots, p_{n}$ of $p$, each equivalent to $q$.

If $p \sim p^{\prime}, q \sim q^{\prime}$, then there is an obvious isometric isomorphism from $p A q$ onto $p^{\prime} A q^{\prime}$ sending $L g_{(p, q)}(A)$ onto $L g_{\left(p^{\prime}, q^{\prime}\right)}(A)$, given by $x \mapsto u x v$, where $u^{*} u=p$, $u u^{*}=p^{\prime}, v v^{*}=q, v^{*} v=q^{\prime}$.

Proposition 3.2. Let $A$ be a $C^{*}$-algebra, and $p, q$ projections in $A$.

(i) There exists a left invertible element in $p A q$ if and only if $q \precsim p$.

(ii) If $\operatorname{sr}(q A q)=n<\infty$ and $n \cdot q \precsim p$, then the left invertible elements of $p A q$ are dense in $p A q$.

(iii) If $p$ is equivalent to a proper subprojection of $q$, then the left invertible elements of $p A q$ are not dense in $p A q$.

Proof. (i) If $u^{*} u=q$ and $u u^{*} \leq p$, then $u \in p A q$ and is left invertible. Conversely, if $x \in p A q$ is left invertible, with $y \in q A p$ with $y x=q$, then $q=x^{*} y^{*} y x \leq\|y\|^{2} x^{*} x$, so $x^{*} x$ is invertible in $q A q$. If $r \in q A q$ with $x^{*} x r=q$, set $u=x r^{1 / 2}$. Then $u^{*} u=q$ and $u u^{*}$ is a projection in $p A p$.

(ii) If $p_{1}, \ldots, p_{n}$ are mutually orthogonal subprojections of $p$, and $u_{i}$ satisfies $u_{i}^{*} u_{i}=p_{i}$ and $u_{i} u_{i}^{*}=q$ for $1 \leq i \leq n$, set $r=p-\sum p_{i}$. If $x \in p A q$, write $x_{i}=p_{i} x$ for $1 \leq i \leq n$ and $x_{n+1}=r x$. Set $y_{i}=u_{i} x_{i} \in q A q$ for $1 \leq i \leq n$. By assumption, $\left(y_{1}, \ldots, y_{n}\right)$ can be approximated by $\left(z_{1}, \ldots, z_{n}\right) \in L g_{n}(q A q)$, i.e. with $\sum_{i=1}^{n} z_{i}^{*} z_{i}$ invertible in $q A q$. Set $w_{i}=u_{i}^{*} z_{i}$ for $1 \leq i \leq n$, and $w=\sum_{i=1}^{n} w_{i}+x_{n+1}$. Then $w$ closely approximates $x$, and $w^{*} w=\sum_{i=1}^{n} z_{i}^{*} z_{i}+x_{n+1}^{*} x_{n+1}$ is invertible in $q A q$, so $w$ is left invertible in $p A q$.

(iii) We may assume $p \supsetneqq q$. Suppose $x=p x q \in p A q$ is left invertible and approximates $p$ closely enough that $x p=p x p$ is invertible in $p A p$. Let $y=q y p \in$ $q A p$ with $y x=q$. If $r=q-p$, then $[r y p][p x p]=\operatorname{ryxp}=(q-p) p=0$, and since $p x p$ is invertible in $p A p, r y p=0$. But $[r y p][p x r]=r y x r=r q r=r \neq 0$, a contradiction.

Part (iii) is a version of the well-known fact that a proper isometry in a (unital) $\mathrm{C}^{*}$-algebra cannot be a limit of invertible elements. See $\mathrm{Rør} 88$ for more detailed results along this line.

Recall that a projection $p$ in a $\mathrm{C}^{*}$-algebra $A$ is infinite if $p$ is equivalent to a proper subprojection of $p$; otherwise $p$ is finite. The projection $p$ is properly infinite if there are subprojections $p_{1}, p_{2}$ of $p$ with $p_{1} \perp p_{2}$ and $p_{1} \sim p_{2} \sim p$. A unital $\mathrm{C}^{*}$-algebra $A$ is finite [infinite, properly infinite] if $1_{A}$ is finite [infinite, properly infinite]. $A$ is finite if and only if every left invertible element of $A$ is invertible.

Corollary 3.3 ([ie83, 3.1,6.5], Rob80]). Let $A$ be a unital $C^{*}$-algebra.

(i) If $\operatorname{sr}(A)=1$, then $A$ is finite.

(ii) If $A$ is properly infinite, then $\operatorname{sr}(A)=\infty$. 
Part (i) follows from 3.2(iii), applied to $p=q=1_{A}$; and (ii) follows from 3.2 (ii)(iii), since if $A$ is properly infinite, $n \cdot 1$ is equivalent to a proper subprojection of 1 for every $n$.

Although a $\mathrm{C}^{*}$-algebra of stable rank 1 must be finite (in fact, stably finite by 4.4), an infinite (unital) $\mathrm{C}^{*}$-algebra need not have infinite stable rank: if $T$ is the Toeplitz algebra, then it is not difficult to show that $\operatorname{sr}(T)=2$ [Rie83, 4.13]. And any stable $\mathrm{C}^{*}$-algebra has stable rank $\leq 2$ [Rie83, 6.4].

\section{EXPANDING AND CONTRACTING MATRICES}

The next two propositions can be used to establish the behavior of stable rank for full corners, as well as obtaining Rieffel's results on the stable rank of matrix algebras. The proofs are adaptations of Rieffel's arguments for the matrix case.

Note that the elements of $p A p$ act on $p A q$ by left multiplication, and left multiplication by an invertible element sends $L g_{(p, q)}(A)$ onto itself.

Recall that an element in a unital ring of the form $1+x, x$ nilpotent, is called unipotent. A unipotent element is invertible.

Proposition 4.1 (cf. [Rie83, 3.4]). Let $A$ be a $C^{*}$-algebra, and $p, q$, and $r$ projections in $A$ with $p \perp r, q \perp r$. If $L g_{(p+r, q+r)}(A)$ is dense in $(p+r) A(q+r)$, then $L g_{(p, q)}(A)$ is dense in $p A q$.

Proof. Let $x \in p A q$. Let $0<\epsilon<1$, and approximate $x+r$ within $\epsilon$ by an element $y \in \operatorname{Lg}_{(p+r, q+r)}(A)$. Then $\|r y q\|<\epsilon,\|p y r\|<\epsilon$, and $\|r-r y r\|<\epsilon$. Thus there is an $a \in r A r$ with $\|a\|<(1-\epsilon)^{-1}$ and $a(r y r)=r$. We then have

$$
(p+r-\text { pyra }) y(q+r-a r y q)=p y q-(\text { pyr }) a(r y q)+r y r .
$$

Also, $p+r-p y r a$ and $q+r-a r y q$ are unipotent and hence invertible in $(p+r) A(p+r)$ and $(q+r) A(q+r)$ respectively, and $y$ is left invertible in $(p+r) A(q+r)$, so pyq- $($ pyr $) a(r y q)+r y r$ is left invertible in $(p+r) A(q+r)$, and hence $p y q-(p y r) a(r y q)$ is left invertible in $p A q$. But

$$
\begin{gathered}
\| x-[p y q-(\text { pyr }) a(r y q)]\|\leq\| x-p y q\|+\| p y r\|\| a\|\| r y q \| \\
<\epsilon+\epsilon^{2}(1-\epsilon)^{-1}=\epsilon(1-\epsilon)^{-1} .
\end{gathered}
$$

Proposition 4.2 (cf. [Rie83, 6.1]). Let $A$ be a $C^{*}$-algebra, and $p, q$, and $r$ projections in $A$ with $p \perp r, q \perp r, r \precsim n \cdot q$ for some $n$. If $L g_{(p, q)}(A)$ is dense in $p A q$, then $\operatorname{Lg}_{(p+r, q+r)}(A)$ is dense in $(p+r) A(q+r)$.

Proof. We may assume $q \leq p$ by 3.2 (i). It suffices to prove the result for $r \sim q$, for then the case where $r \sim\left(2^{n}-1\right) \cdot q$ follows by induction, and 4.1 then gives the case $r \precsim n \cdot q$. Since $s=p-q+r \sim p, L g_{(s, q)}(A)$ and $L g_{(s, r)}(A)$ are dense in $s A q$ and $s A r$ respectively. If $x \in(p+r) A(q+r)$ and $\epsilon>0$, then $x$ can be approximated within $\epsilon / 2$ by $y \in(p+r) A(q+r)$ such that $a=s y q \in L g_{(s, q)}(A)$. We will show there is an invertible $z \in(p+r) A(p+r)$ such that $z y q=q$. This will suffice to prove the statement, since by hypothesis there will be $w \in L g_{(s, r)}(A)$ approximating szyr within $\epsilon / 2\left\|z^{-1}\right\|$, and then $q+w+q z y r$ will be an element of $L g_{(p+r, q+r)}(A)$ approximating $q+s z y r+q z y r=z y q+(p+r) z y r=z y$ within $\epsilon / 2\left\|z^{-1}\right\|$, so $z^{-1}(q+w+q z y r) \in L g_{(p+r, q+r)}(A)$ approximates $y$ within $\epsilon / 2$ and therefore approximates $x$ within $\epsilon$. 
To find $z$, first note that if $b \in q A s$ with $b a=q$, then $u=p+r+(q-q y q) b$ is unipotent and hence invertible in $(p+r) A(p+r)$; if $v=p+r-s y q$, then $v$ is unipotent in $(p+r) A(p+r)$ and $v u y q=q$. Set $z=v u$.

The proofs of Propositions 4.1 and 4.2 are easier to follow and understand if elements are written symbolically as matrices, as described earlier.

Note that Proposition 4.2 is not true in general if $r$ is "too large" compared to $q$ : let $\mathcal{H}$ be an infinite-dimensional Hilbert space, $A=\mathcal{B}(\mathcal{H}), p=q$ a finite-rank projection, and $r=1-p$.

Corollary 4.3. Let $A$ be a unital $C^{*}$-algebra and $m \geq 0$. If the left invertible $(m+1) \times 1$ matrices are dense in the $(m+1) \times 1$ matrices over $A$, then the left invertible $(m+k) \times k$ matrices are dense in the $(m+k) \times k$ matrices over $A$ for all $k$. Conversely, if the left invertible $(m+k) \times k$ matrices are dense for some $k$, then the left invertible $(m+1) \times 1$ matrices over $A$ are dense in $A^{m+1}$.

Proof. Apply the previous two propositions to $M_{m+k}(A)$ with $p, q$, and $r$ diagonal projections of rank $m+1,1$, and $k-1$ respectively.

Corollary 4.4 ([Rie83, 6.1]). Let $A$ be a $C^{*}$-algebra. Then, for any $n$, we have $\operatorname{sr}\left(M_{n}(A)\right)=\left\lceil\frac{\operatorname{sr}(A)-1}{n}\right]+1$, where $\lceil\cdot\rceil$ denotes "least integer $\geq$." In particular, $\operatorname{sr}\left(M_{n}(A)\right)=1$ [resp. $\infty$ ] if and only if $\operatorname{sr}(A)=1$ [resp. $\infty$ ].

Indeed, the $r \times 1$ matrices over $M_{n}(A)$ can be identified with the $n r \times n$ matrices over $A$. Thus, if $\operatorname{sr}(A)=m+1, \operatorname{Lg}_{r}\left(M_{n}(A)\right)$ is dense in $\left(M_{n}(A)\right)^{r}$ if and only if $m+n \leq n r$. (In the nonunital case, one must also show that $\operatorname{sr}\left(M_{n}(\tilde{A})\right)=$ $\operatorname{sr}\left(\widehat{M_{n}(A)}\right)$; cf. [Rie83, 6.1].)

Recall that a projection $p$ in a $\mathrm{C}^{*}$-algebra $A$ is full if it is not contained in any proper closed, two-sided ideal of $A$. If $A$ is unital and $p$ is full in $A$, then $1 \precsim n \cdot p$ for some $n$, in some matrix algebra over $A$ Cun77.

The main result of this article is another consequence of Proposition 4.2:

Theorem 4.5. Let $A$ be a unital $C^{*}$-algebra and $p$ a full projection in $A$. Then $\operatorname{sr}(p A p) \geq \operatorname{sr}(A)$. If $\operatorname{sr}(A)<\infty$, then $\operatorname{sr}(p A p)<\infty$; and $\operatorname{sr}(p A p)=1$ if and only if $\operatorname{sr}(A)=1$.

Proof. We have that $1 \precsim n \cdot p$ for some $n$, and hence $1-p \precsim n \cdot p$. If $m=$ $s r(p A p)<\infty$, then $L g_{(m \cdot p, p)}(A)$ is dense in $(p A p)^{m}$, and hence by Proposition $4.2 \operatorname{Lg}_{(m \cdot p+1-p, p+1-p)}(A)$ is dense in the corresponding column space, which may be regarded as a subspace of either $A^{m}$ or $M_{m}(A)$ (1 denotes the identity of $A$ ). The same is then true for $L g_{(m \cdot p+m \cdot(1-p), 1)}(A) \cong L g_{m}(A)$ in $A^{m}$, i.e. $\operatorname{sr}(A) \leq m$. On the other hand, 1 is equivalent to a full projection in $M_{n}(p A p)$, so $\operatorname{sr}(A) \geq$ $\operatorname{sr}\left(M_{n}(p A p)\right)=\left\lceil\frac{s r(p A p)-1}{n}\right]+1$; in particular, if $\operatorname{sr}(p A p)=\infty$, then $\operatorname{sr}(A)=\infty$. Also, if $\operatorname{sr}(p A p)=1$, then $\operatorname{sr}(A) \leq \operatorname{sr}(p A p)=1$, and conversely if $\operatorname{sr}(A)=1$, then $\operatorname{sr}\left(M_{n}(p A p)\right) \leq \operatorname{sr}(A)=1$, and hence $\operatorname{sr}(p A p)=1$ by Corollary 4.4.

Simple counterexamples show that the hypothesis that $p$ be full is necessary: for example, $A=\mathcal{B}(\mathcal{H})$ for infinite-dimensional $\mathcal{H}, p$ a finite-rank projection; or $A=\mathbb{C} \oplus O_{2}, p=(1,0)$. 
What about the nonunital case? It is very plausible that if $p$ is a full projection in $A$, then $\operatorname{sr}(p A p) \geq \operatorname{sr}(A)$ even if $A$ is nonunital; however, this does not seem to follow from our arguments except in a special case:

Corollary 4.6. Let $A$ be a $C^{*}$-algebra with an approximate unit $\left(q_{i}\right)$ of projections, and let $p$ be a full projection in $A$. Then $\operatorname{sr}(p A p) \geq \operatorname{sr}(A)$.

Proof. We have $\operatorname{sr}(A) \leq \liminf \operatorname{sr}\left(q_{i} A q_{i}\right)$, and there is an $i_{0}$ such that $p \precsim q_{i}$ for all $i \geq i_{0}$, so $\operatorname{sr}(p A p) \geq \operatorname{sr}\left(q_{i} A q_{i}\right)$ for $i \geq i_{0}$.

It is less clear whether a general full corner in a nonunital $\mathrm{C}^{*}$-algebra $A$ (a hereditary $\mathrm{C}^{*}$-subalgebra of the form $p A p$ for a projection $p$ in the multiplier algebra $M(A)$, with $\operatorname{span}(A p A)$ dense in $A$ ) also satisfies the inequality. As pointed out by $\mathrm{N}$. Elhage Hassan, the inequality fails for general full hereditary $\mathrm{C}^{*}$-subalgebras: if $A$ is a purely infinite simple unital $\mathrm{C}^{*}$-algebra, e.g. $O_{2}$, and $B$ is a nonunital hereditary $\mathrm{C}^{*}$-subalgebra, then $B$ is stable, so $2=\operatorname{sr}(B)<\operatorname{sr}(A)=\infty$.

\section{REFERENCES}

[Bas68] Hyman Bass, Algebraic K-theory, W. A. Benjamin, Inc., New York-Amsterdam, 1968. MR 40:2736

[Bla83] Bruce Blackadar, A stable cancellation theorem for simple $C^{*}$-algebras, Proc. London Math. Soc. (3) 47 (1983), no. 2, 303-305, Appendix to: "The cancellation theorem for projective modules over irrational rotation $C^{*}$-algebras" [Proc. London Math. Soc. (3) 47 (1983), no. 2, 285-302] by M. A. Rieffel. MR 85g:46086

[BP91] Lawrence G. Brown and Gert K. Pedersen, $C^{*}$-algebras of real rank zero, J. Funct. Anal. 99 (1991), no. 1, 131-149. MR 92m:46086

[Cun77] Joachim Cuntz, The structure of multiplication and addition in simple $C^{*}$-algebras, Math. Scand. 40 (1977), no. 2, 215-233. MR 58:17862

[GJ60] Leonard Gillman and Meyer Jerison, Rings of continuous functions, The University Series in Higher Mathematics, D. Van Nostrand Co., Inc., Princeton, N.J.-Toronto-London-New York, 1960. MR 22:6994

[HV84] Richard H. Herman and Leonid N. Vaserstein, The stable range of $C^{*}$-algebras, Invent. Math. 77 (1984), no. 3, 553-555. MR 86a:46074

[HW41] Witold Hurewicz and Henry Wallman, Dimension Theory, Princeton Mathematical Series, v. 4, Princeton University Press, Princeton, N. J., 1941. MR 3:312b

[Kat50] Miroslav Katětov, On rings of continuous functions and the dimension of compact spaces, Časopis Pěst. Mat. Fys. 75 (1950), 1-16. MR 12:119b

[Pea75] A. R. Pears, Dimension theory of general spaces, Cambridge University Press, Cambridge, England, 1975. MR 52:15405

[Rie83] Marc A. Rieffel, Dimension and stable rank in the $K$-theory of $C^{*}$-algebras, Proc. London Math. Soc. (3) 46 (1983), no. 2, 301-333. MR 84g:46085

[Rob80] A. Guyan Robertson, Stable range in $C^{*}$-algebras, Math. Proc. Cambridge Philos. Soc. 87 (1980), no. 3, 413-418. MR 82h:46079

[Rør88] Mikael Rørdam, Advances in the theory of unitary rank and regular approximation, Ann. of Math. (2) 128 (1988), no. 1, 153-172. MR 90c:46072

[Vn71] L. N. Vaseršteĭn, The stable range of rings and the dimension of topological spaces, Funkcional. Anal. i Priložen. 5 (1971), no. 2, 17-27. MR 44:1701

[War80] R. B. Warfield, Jr., Cancellation of modules and groups and stable range of endomorphism rings, Pacific J. Math. 91 (1980), no. 2, 457-485. MR 82m:16022

[Win01] Wilhelm Winter, Covering dimension for nuclear $C^{*}$-algebras $i$, ii, Preprint, 2001. MR 2002c: 46134

Department of Mathematics/084, University of Nevada, Reno, Reno, Nevada 89557

E-mail address: bruceb@math.unr.edu 